\title{
Exact Output Response Computation of RC Interconnects Under Polynomial Input Waveforms
}

\author{
Satrajit Gupta \\ Indian Institute of Science \\ Dept. of Electrical Communication Engineering \\ Bangalore,India \\ satrajit@protocol.ece.iisc.ernet.in
}

\author{
L.M.Patnaik \\ Indian Institute of Science \\ Microprocessor Applications Laboratory \\ Bangalore,India \\ lalit@micro.iisc.ernet.in
}

\begin{abstract}
Accurate output response computation of $R C$ interconnects under various input excitations is a key issue in deep submicron delay analysis.In this paper,we present an exact analysis of output response computation of a distributed $R C$ interconnect under input signals that are polynomial in time $\left(t^{n}\right)$.A simple, recursive equation that helps us to calculate the interconnect response under higher order polynomial inputs in terms of the lower order polynomial responses is derived.To the best of our knowledge, this is the first exact output response analysis of $R C$ interconnects under generalized polynomial inputs.
\end{abstract}

\section{Introduction}

A major concern in state-of-the-art deep submicron design is to accurately model and characterize the interconnect delay.Traditionally Elmore delay[3] is used as a metric for estimating delay through RC interconnects. However,it is at best an upper bound on RC interconnect delay.And although researchers have proved Elmore delay to be a bound on the 50\% threshold delay for RC interconnects under input waveforms more general than step[4], it has been reported that Elmore delay can be very different from Spicecomputed 50\% delay.In fact depending on the input slew time and driver resistance,the Elmore delay can be either greater or less than the Spice-computed delay[5]. This has prompted researchers to look for more accurate and computationally efficient delay estimates through $\mathrm{RC}$ interconnects.

In this paper,we present analytical expressions for output response of RC interconnects under input waveforms that are polynomial in time.It is shown that a recursive formula that helps us to calculate the output response for higher order input polynomial waveforms from the output response expression for lower order input polynomial waveforms can be derived.Once we get the output reponse expression, the delay at a particular threshold voltage (50\% or $90 \%$ ) can be obtained easily by numerical or graphical methods.

The paper is organized as follows.In section 2 we present a survey of the work on RC interconnets. The output response computation formulae are presented in section 3.Finally section 4 concludes with a discussion on the future scope of the work.

\section{RC Interconnect Analysis -A Survey}

Broadly, the study of RC interconnects can be divided into two categories

- Accurate time domain or s-domain analysis of the interconnect structure which yields an analytic expression of the output voltage waveform. This is the method we will be concentrating on in our analysis.

- Moment-matching methods for RC interconnect transfer function synthesis where the RC interconnect transfer function is modelled as a reduced order rational function in s-domain. The rational function coefficients are obtained by moment-matching.

A complete time-domain solution of the RC interconnect was given in Kaufman and Garrett[8]. However they made the simplifying assumption of separability which means that the voltage response function can be decomposed into separate functions of time and position. Recently Kahng and Muddu gave exact expression for output response of a semi-infinite RC line under step input[7].They extended their work to include ramp input in a subsequent paper[9] where they presented a general approach to compute the time-domain ramp response of finite RC lines.They gave closed form expresions of ramp input response for both open-ended and capacitively loaded finite RC lines.Another interesting work in this area is that of Rao[10]. 


\subsection{Limitations of the previous approaches}

However, all these approaches deal with a very restricted class of inputs-step and ramp.Real-life waveforms in VLSI circuits can be very different from these simple abstractions.This difference between real-life waveforms and the waveforms used for output response computation can be crucial for deep submicron delay estimation.Specifically,it has been shown that because of increasing interconnect resistance, in state-of-the-art designs the driver sees only a part of the total net capacitance. This phenomenon is referred to as 'resistance shielding'[6]. Because of this, the waveform at the input of the interconnect shows a nonlinear behaviour and this can strongly influence the delay of the RC interconnect.

This has been the motivation for us to look for analytic expressions of RC interconnect outputs under more general input waveforms than step or ramp. With this in mind, we present analytical expressions of distributed semi-infinite $\mathrm{RC}$ interconnect response under input waveforms that are polynomial in time $\left(t^{n}\right)$. The reason behind choosing a polynomial waveform is the fact that any analytic function in its radius of convergence can be represented as a power series which consists of a summation of an infinite number of polynomials.Also,we can fit an arbitrary waveform with a polynomial expression. So once we have analytic expressions for $t^{n}$ type of polynomial inputs we can approximate the response to an arbitrary waveform by summing the appropriate polynomial $\left(t^{n}\right)$ responses. This serves as a more powerful framework for considering generalized input waveforms.In fact there have been non-linear waveform characterizations already in the literature[11].In the next section we present the details of our analysis.

\section{Analysis under Polynomial Input}

Now we present the time-domain response of RC interconnects under polynomial input waveforms. We assume the following form for an $n$-th order infinite input polynomial waveform,

$$
V_{i n}(t)=\frac{V_{0}}{T_{R}^{n}} t^{n} u(t)
$$

where $T_{R}$ is the time at which the input waveform reaches the value $V_{0}$ and $u(t)$ is the unit step waveform that starts at time $t=0$.

We consider the case of the semi-infinite RC line first.

The diffusion equation for a distributed RC line is given by,

$$
\frac{\partial^{2} v_{n}(x, t)}{\partial x^{2}}=r c \frac{\partial v_{n}(x, t)}{\partial t}
$$

where $r$ and $c$ are the per unit length resistance and capacitance respectively of the interconnect and $v_{n}(x, t)$ is the output voltage waveform at a distance $x$ from the source at time $t$ under $n$-th order infinite polynomial input(i. e. a polynomial input of the form $\frac{V_{0}}{T_{R}^{n}} t^{n} u(t)$ as defined earlier). The boundary conditions for the problem are:

$$
\begin{aligned}
& v_{n}(x, 0)=0 \\
& v_{n}(0, t)=V_{i n}(t)=\frac{V_{0}}{T_{R}^{n}} t^{n} u(t)
\end{aligned}
$$

We denote

$$
w_{n, k}(x, t)=\frac{\partial^{k} v_{n}(x, t)}{\partial t^{k}} \text { for } k=0, \ldots, n
$$

Clearly $w_{n, 0}(x, t)=v_{n}(x, t)$ and $w_{n, n}(x, t)=\frac{\partial^{n} v_{n}(x, t)}{\partial t^{n}}$. Now the problem can be transformed into:

$$
\frac{\partial^{2} w_{n, n}(x, t)}{\partial x^{2}}=r c \frac{\partial w_{n, n}(x, t)}{\partial t}
$$

With the boundary conditions:

$$
\begin{aligned}
& w_{n, n}(x, 0)=0 \quad \text { for all } \mathrm{x} \geq 0(2) \\
& w_{n, n}(0, t)=\frac{n ! V_{0}}{T_{R}^{n}} u(t) \text { for all } \mathrm{t} \geq 0(3)
\end{aligned}
$$

The solution to the transformed problem is given by[7]:

$$
w_{n, n}(x, t)=\frac{n ! V_{0}}{T_{R}^{n}} \operatorname{erfc}\left(\frac{x \sqrt{r c}}{\sqrt{4 t}}\right)
$$

Let us denote $V_{0} \operatorname{erfc}\left(\frac{x \sqrt{r c}}{\sqrt{4 t}}\right)=I_{0}(x, t)$ and let $I_{i+1}(x, t)=$ $\int_{0}^{t} I_{i}(x, \tau) d \tau$ for $\mathrm{i}=0,1,2, \ldots$

Then $v_{n}(x, t)$ is given by:

$$
v_{n}(x, t)=\frac{n !}{T_{R}^{n}} I_{n}(x, t)
$$

It can be shown that

$$
I_{2}(x, t)=\left(k_{1,2} t+k_{2,2}\right) I_{1}(x, t)-k_{3,2} t^{2} I_{0}(x, t)
$$

where $k_{1,2}=5 / 6, k_{2,2}=\frac{x^{2} r c}{12}, k_{3,2}=1 / 3$

Further,it can be easily shown that,

if

$I_{m}(x, t)=\left(k_{1, m} t+k_{2, m}\right) I_{m-1}(x, t)-k_{3, m} t^{2} I_{m-2}(x, t)$

then

$I_{m+1}(x, t)=\left(k_{1, m+1} t+k_{2, m+1}\right) I_{m}(x, t)-k_{3, m+1} t^{2} I_{m-1}(x, t)$

where

$$
\begin{aligned}
& k_{1, m+1}=\left(\frac{k_{1, m}-2 k_{3, m}}{1+k_{1, m}-2 k_{3, m}}\right) \\
& k_{2, m+1}=\left(\frac{k_{2, m}}{1+k_{1, m}-2 k_{3, m}}\right) \\
& k_{3, m+1}=\left(\frac{k_{3, m}}{1+k_{1, m}-2 k_{3, m}}\right)
\end{aligned}
$$


Using equations (7) and (8) we can recursively compute $I_{k}(x, t)$ for $k=3,4, \ldots$

Now,the response of a semi-infinite RC line under a $(n+$ 1)st polynomial $\left(\frac{V_{0}}{T_{R}^{n+1}} t^{n+1} u(t)\right)$ is given by,

$$
v_{n+1}(x, t)=\frac{(n+1) !}{T_{R}^{n+1}} I_{n+1}(x, t)
$$

We substitute $m=n$ in equation (8) to get,

$$
I_{n+1}(x, t)=\left(k_{1, n+1} t+k_{2, n+1}\right) I_{n}(x, t)-k_{3, n+1} t^{2} I_{n-1}(x, t)
$$

For an $m$-th order input waveform the response is given by,

$$
v_{m}(x, t)=\frac{m !}{T_{R}^{m}} I_{m}(x, t)
$$

From equations (9),(10) and (11) we get,

$$
\begin{aligned}
v_{n+1}(x, t)= & \frac{(n+1)}{T_{R}}\left[\left(k_{1, n+1} t+k_{2, n+1}\right) v_{n}(x, t)-\right. \\
& \left.\frac{n k_{3, n+1}}{T_{R}} t^{2} v_{n-1}(x, t)\right]
\end{aligned}
$$

where $k_{1, n+1}, k_{2, n+1}$ and $k_{3, n+1}$ are as defined earlier. We can see from equation (12) that the response for the $(n+1)$ st order polynomial input can be derived in terms of responses for the $n$-th order and $(n-1)$ st order polynomials. Since we have closed form solutions for responses to step,ramp[9] and quadratic input waveforms(6), we can recursively generate exact expressions of higher order polynomial response waveforms.

Till now we have considered infinite polynomial input waveforms.Now we can derive the response for the more practical case of finite polynomial input waveforms (i. e. polynomial input waveforms that saturate to a fixed value after a finite amount of time).

We assume the finite polynomial input to saturate at a value $V_{0}$ after time $T_{R}$.

The input waveform expression in this case is given by:

$$
\begin{aligned}
V_{i n}(t)= & \frac{V_{0}}{T_{R}^{n}}\left[t^{n} u(t)-\left(t^{n}-T_{R}^{n}\right) u\left(t-T_{R}\right)\right] \\
= & \frac{V_{0}}{T_{R}^{n}}\left[t^{n} u(t)-\right. \\
& \left.\sum_{k=1}^{n}{ }^{n} C_{k} T_{R}^{n-k}\left(t-T_{R}\right)^{k} u\left(t-T_{R}\right)\right]
\end{aligned}
$$

Let us denote the output response in the case of a finite polynomial input by $u_{n}(x, t)$

From equations $(11),(12)$ and (14) we see that $u_{n}(x, t)$ is given by,

$$
u_{n}(x, t)=v_{n}(x, t)-\sum_{k=1}^{n}{ }^{n} C_{k} v_{k}\left(x, t-T_{R}\right)
$$

where $v_{k}(x, t)$ is the output response to the infinite polynomial input $\frac{V_{0}}{T_{R}^{k}} t^{k} u(t)$.It can be easily calculated using the iterative equation (12) given above.

With equation no. (15), we can derive the exact timedomain response of an RC interconnect under finite polynomial input waveform.

As mentioned before,we can represent any arbitrary waveform with an analytic representation as a power series.For practical purposes, we can truncate the series after a suitable number of polynomial terms. We can find the response separately for each polynomial term using the equations (12) and (15) given above and sum the responses to get the final response waveform.

\subsection{Extension to the case of finite RC lines}

Till now we have analyzed the response of a semi-infinite $\mathrm{RC}$ line under polynomial input waveforms. When we consider the practical case of a finite RC line with arbitrary load impedance,the situation becomes different because of the load and source reflections. As a result,the voltage waveform at $(\mathrm{x}, \mathrm{t})$ is a sum of the incident waveform and an infinite number of reflected waveforms.

Let $\Gamma_{l}(s)$ and $\Gamma_{s}(s)$ be the s-domain reflection coefficients at the source and the load ends respectively.Then

$$
\text { and } \quad \begin{aligned}
\Gamma_{l}(s) & =\frac{Z_{l}-Z_{o}}{Z_{l}+Z_{o}} \\
\Gamma_{s}(s) & =\frac{Z_{s}-Z_{o}}{Z_{s}+Z_{o}}
\end{aligned}
$$

where $Z_{l}(s), Z_{s}(s)$ and $Z_{o}(s)$ denote the load,source and characteristic impedance of the line respectively.

In this case the expression for the output waveform in s-domain is given by[9]

$$
\begin{aligned}
U_{n}(x, s)= & V_{n}(x, s)+\sum_{k=1}^{\infty}\left[\Gamma_{l}^{k}(s) \Gamma_{s}^{k-1}(s) V_{n}(2 k L-x, s)+\right. \\
& \left.\Gamma_{l}^{k}(s) \Gamma_{s}^{k}(s) V_{n}(2 k L+x, s)\right]
\end{aligned}
$$

where $U_{n}(x, s)$ is the s-domain output response at distance $x$ from the source, $V_{n}(x, s)$ is the s-domain incident waveform, and $L$ is the length of the finite interconnect.The suffix $n$ indicates that the input is an $n$-th order polynomial of the form $\frac{V_{0}}{T_{R}^{n}}\left[t^{n} u(t)\right]$.The incident waveform is the Laplace transform of the time domain response of a semi-infinite $\mathrm{RC}$ line under $n$-th order polynomial input.This can be obtained using equations (12) and (15) derived in the last subsection.

The special case of a finite open-ended RC line with ideal source can be easily analyzed.In this case we have, $Z_{s}(s)=$ $0, Z_{l}(s)=\infty$ and so $\Gamma_{l}(s)=1, \Gamma_{s}(s)=-1$. 
Therefore from equation (16) we have,

$$
\begin{aligned}
U_{n}(x, s)= & V_{n}(x, s)+\sum_{k=1}^{\infty}\left[(-1)^{k-1} V_{n}(2 k L-x, s)+\right. \\
& \left.(-1)^{k} V_{n}(2 k L+x, s)\right]
\end{aligned}
$$

The corresponding time-domain expression is given by,

$$
\begin{aligned}
u_{n}(x, t)= & v_{n}(x, t)+\sum_{k=1}^{\infty}\left[(-1)^{k-1} v_{n}(2 k L-x, t)+\right. \\
& \left.(-1)^{k} v_{n}(2 k L+x, t)\right]
\end{aligned}
$$

where $u_{n}(x, t)$ and $v_{n}(x, t)$ are the time domain finite and semi-infinite RC line responses respectively to $n$-th order input polynomial waveform.

As is evident from equation (18), we can directly use the results derived in the last subsection and obtain an expression for the case of finite RC line.

\section{Conclusions}

In this paper we presented analytical expressions for output voltage response of semi-infinite RC lines under polynomial input waveforms. We showed that the output response of a RC interconnect to an $n$-th order polynomial is obtained in terms of the responses to $(n-1)$ st order and $(n-2)$ nd order polynomials. Since the output responses of an RC line for step,ramp and quadratic inputs are easy to derive, these responses can be used to recursively compute the response to an n-th order polynomial. This provides us with a framework to estimate delays of interconnect structures to input waveforms more general than step and ramp.This is important,because in deep sub micron designs input waveshape can play a role in delay estimation.

There is a lot of scope for future work in this area. We need to come up with high-fidelity polynomial approximations to real-life waveforms. This can be done by defining empirical parameters that capture the waveshape of a given waveform faithfully.Then each waveform can be represented by a unique set of these parameters and we can come up with a polynomial fit specific to a waveform by using these parameters. We can feed this polynomial approximation to the RC interconnect and estimate the output response and delay using the equations derived in the last section. This procedure can be extended to RC trees and we can approximate the waveform at each discontinuity in the tree with polynomial expressions using the parameter set. In this way we can generate exact output response at all the nodes of a circuit.

\section{References}

[1] H.B.Bakoglu, "Circuits,Interconnections and Packaging", Addison Wesley,1990
[2] K.C.Saraswat and F.Mohammadi,"Effect of scaling of interconnections on the time delay of VLSI circuits",IEEE J.Solid-State Circuits SC-17(2),April 1982, pp. 275-280.

[3] W.C.Elmore,"The transient response of damped linear network with particular regard to wideband amplifiers",J.App.Physics 19,Jan.1948,pp. 55-63.

[4] R.Gupta et al.,"The Elmore delay as a bound for RC trees with generalized input signals",ACM/IEEE Design Automation Conference,June 1995,pp. 364-369

[5] A.B.Kahng et al,"Analytical Delay Models for VLSI Interconnects Under Ramp Input”, ICCAD,Nov 1996,pp. 30-35.

[6] J.Qian et al.,"Modelling the effective capacitance for the RC interconnect of CMOS trees",IEEE Trans. on CAD,13(12),Dec.1994,pp.1526-1535

[7] A.B.Kahng and S.Muddu,"Delay analysis of VLSI interconnections using the diffusion equation model",IEEE Design Automation Conference,June 1994,pp. 563-569

[8] W.M.Kaufman and S.J.Garrett,"Tapered distributed filters",IRE Trans. on Circuit Theory,Dec.1962,pp.542-543

[9] A.B.Kahng and S.Muddu,"Analysis of RC interconnects under ramp input”, ACM TODAES 2(2),April 1997,pp.168192.

[10] V.B.Rao,"Delay analysis of the distributed RC line", ACM/IEEE Design Automation Conference,June 1995, pp.370-375.

[11] D.Holberg et al,"DC parameterized piecewise function transistor models for bipolar and MOS logic stage delay evaluation”,ICCAD,Nov.1990,pp. 546-549. 